\title{
Dynamic Analysis of the Driving System of the Shearer's Cutting Unit Based on the Transfer Matrix Method
}

\author{
Hong-bin GAO ${ }^{1, a^{*}}$ and Gai-lin LU ${ }^{1, b}$ \\ ${ }^{1}$ Shanxi University, China \\ aghb9420@163.com, ${ }^{b} 756533342 @ q q . c o m$
}

Keywords: Transfer matrix method; Shearer; Driving system

Abstract.For further research on roller transmission system dynamics characteristics of the coal winn ing machine cutting part, structures of the drive system are analyzed in detail, which is divided into th e fixed axis gear train and planetary gear train two parts to analysis and research. Taking a certain ty pe of drum shearer cutting part transmission system as an example,to simplify model for centralized mass mechanics of gear shaft system. with the transfer matrix method as the theoretical basis, we can obtain the mathematical model of the planetary gear and the fixed axis gear train in the drum of the $r$ ocker arm. The dynamic mathematical model of the transmission system of the shearer cutting part of the drum is obtained by the transfer matrix method by alternative intermediate variable finally.Based on this model, the simulation of the starting process is carried out.The model can provide a theoretic al basis for the prevention and monitoring of the cutting part of coal mining machine and the optimiz ation design.

\section{Background of this research}

The cutting unit of the shearer need undertake a large cutting resistance, which will varies with time and space. The change of the resistance can result in a sudden alteration of the driving system, and lead to motor's sudden acceleration, deceleration, or shutdown ${ }^{[1-4]}$. These factors further intensify connecting vibration fault of the driving system ${ }^{[5-6]}$. In this paper, the dynamic characteristic of the driving system of the shearer's cutting unit will be analyzed based on the transfer matrix method.

\section{The system structure of the driving system}

Taking some shearer as an example, the transmission system structure diagram as shown in Figure 1 of the cutting section. In this drive system, each shafting is a high speed motorized spindle rotor bearing system which is supported by a rolling bearing. Effect of each shafting is rotated by torque

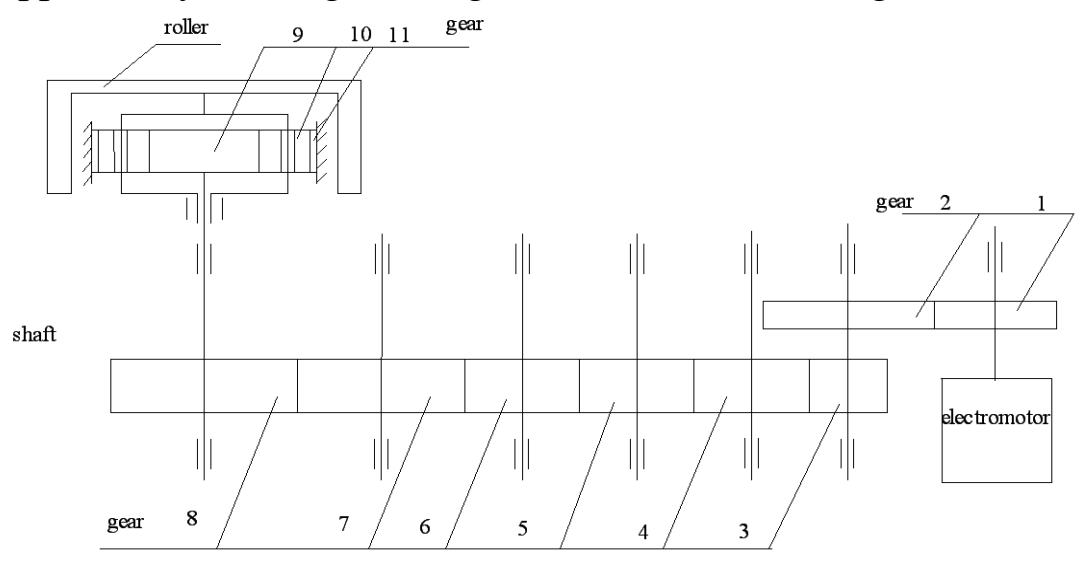

Fig.1 Driving system of the cutting unit

which will be energy from the motor final pass the drum. And complete the coal cutting work. So the dynamic Eq. 1 of the forced rotor bearing system can describe the transmission process ${ }^{[7]}$,

$$
[J](\ddot{\theta})+[C](\dot{\theta})+[K](\theta)=[T]
$$


In Eq. 1, J, $\theta, C, K, T$ is inertia matrix of shaft mass concentration, twist angle matrix in the quality concentration of shafting, shafting damping matrix sets, the shafting stiffness matrix set, and each concentrate quality department of moment vector.

To solve the eigenvalues and eigenvectors of Eq. 1, the dynamic parameters characteristic, natural frequencies and mode shapes, can be obtained. But it is not easy to solve the above equation directly. In this paper, the motion is obtained by using Matlab/Simulink as tool. In the process of establishing the mathematical model, the cutting part of shafting parts are simplified ${ }^{[8]}$ : the gear of each shaft is simplified as a concentrated moment of inertia which is only present in mass and neglects the elasticity $\mathrm{J}_{\mathrm{s}}$, the drive shaft is simplified as an elastic member with no mass and only a torsion stiffness (K)is present, the mass of each shaft is equivalent to the gear; The simplified equivalent of the friction resistance and the bearing is viscous damping $\left(\mathrm{C}_{\mathrm{s}}\right)$ being proportion to the speed ; The stiffness is neglected; The damping caused by the structure and shaft is not considered. The transmission system of the cutting part is composed of a rocker arm deceleration system and a planetary gear reduction system $^{[8]}$, because these two kinds are difference, so this will be the first to establish the two sub model, then the cutting part of the drive system dynamics model.

\section{The mathematical model of the rocker deceleration system}

As shown in Figure 1, shearer rocker reducer is a typical fixed axis gear train, in it there are 7 drive shafts and 8 gears, consider the input torque of the motor and the output torque of the gear 9 , there are 10 moment inertia of mass, and the dynamic mathematical Eq. 2 is established:

$$
\begin{aligned}
& \left(\begin{array}{cccccccccc}
J_{g} & 0 & 0 & 0 & 0 & 0 & 0 & 0 & 0 & 0 \\
0 & J_{1} & 0 & 0 & 0 & 0 & 0 & 0 & 0 & 0 \\
0 & 0 & J_{2} & 0 & 0 & 0 & 0 & 0 & 0 & 0 \\
0 & 0 & 0 & J_{3} & 0 & 0 & 0 & 0 & 0 & 0 \\
0 & 0 & 0 & 0 & J_{4} & 0 & 0 & 0 & 0 & 0 \\
0 & 0 & 0 & 0 & 0 & J_{5} & 0 & 0 & 0 & 0 \\
0 & 0 & 0 & 0 & 0 & 0 & J_{6} & 0 & 0 & 0 \\
0 & 0 & 0 & 0 & 0 & 0 & 0 & J_{7} & 0 & 0 \\
0 & 0 & 0 & 0 & 0 & 0 & 0 & 0 & J_{8} & 0 \\
0 & 0 & 0 & 0 & 0 & 0 & 0 & 0 & 0 & J_{9}
\end{array}\right)\left(\begin{array}{l}
\ddot{\theta_{4}} \\
\ddot{\theta_{5}} \\
\ddot{\theta_{3}} \\
\ddot{\theta_{6}} \\
\ddot{\theta_{2}} \\
\ddot{\theta_{7}} \\
\ddot{\theta_{8}} \\
\ddot{\theta_{9}}
\end{array}\right)+\left(\begin{array}{cccccccccc}
C_{g} & 0 & 0 & 0 & 0 & 0 & 0 & 0 & 0 & 0 \\
0 & C_{1} & 0 & 0 & 0 & 0 & 0 & 0 & 0 & 0 \\
0 & 0 & C_{2} & 0 & 0 & 0 & 0 & 0 & 0 & 0 \\
0 & 0 & 0 & C_{3} & 0 & 0 & 0 & 0 & 0 & 0 \\
0 & 0 & 0 & 0 & C_{4} & 0 & 0 & 0 & 0 & 0 \\
0 & 0 & 0 & 0 & 0 & C_{5} & 0 & 0 & 0 & 0 \\
0 & 0 & 0 & 0 & 0 & 0 & C_{6} & 0 & 0 & 0 \\
0 & 0 & 0 & 0 & 0 & 0 & 0 & C_{7} & 0 & 0 \\
0 & 0 & 0 & 0 & 0 & 0 & 0 & 0 & C_{8} & 0 \\
0 & 0 & 0 & 0 & 0 & 0 & 0 & 0 & 0 & C_{9}
\end{array}\right)\left(\begin{array}{l}
\dot{\theta_{g}} \\
\dot{\theta_{1}} \\
\dot{\theta_{2}} \\
\dot{\theta_{3}} \\
\dot{\theta_{4}} \\
\dot{\theta_{5}} \\
\dot{\theta_{6}} \\
\dot{\theta_{7}} \\
\dot{\theta_{8}} \\
\dot{\theta_{9}}
\end{array}\right) \\
& +\left(\begin{array}{cccccccccc}
K_{g 1} & -K_{g 1} & 0 & 0 & 0 & 0 & 0 & 0 & 0 & 0 \\
-K_{g 1} & K_{g 1} & 0 & 0 & 0 & 0 & 0 & 0 & 0 & 0 \\
0 & 0 & K_{23} & -K_{23} & 0 & 0 & 0 & 0 & 0 & 0 \\
0 & 0 & -K_{23} & K_{23} & 0 & 0 & 0 & 0 & 0 & 0 \\
0 & 0 & 0 & 0 & 0 & 0 & 0 & 0 & 0 & 0 \\
0 & 0 & 0 & 0 & 0 & 0 & 0 & 0 & 0 & 0 \\
0 & 0 & 0 & 0 & 0 & 0 & 0 & 0 & 0 & 0 \\
0 & 0 & 0 & 0 & 0 & 0 & 0 & 0 & 0 & 0 \\
0 & 0 & 0 & 0 & 0 & 0 & 0 & 0 & K_{89} & -K_{89} \\
0 & 0 & 0 & 0 & 0 & 0 & 0 & 0 & -K_{89} & K_{89}
\end{array}\right)\left(\begin{array}{c}
\theta_{g} \\
\theta_{1} \\
\theta_{2} \\
\theta_{3} \\
\theta_{4} \\
\theta_{5} \\
\theta_{6} \\
\theta_{7} \\
\theta_{8} \\
\theta_{9}
\end{array}\right)=\left(\begin{array}{c}
T_{g} \\
T_{21} \\
T_{12} \\
T_{43} \\
T_{34}+T_{54} \\
T_{45}+T_{65} \\
T_{56}+T_{76} \\
T_{87}+T_{67} \\
T_{78} \\
T_{9}
\end{array}\right)
\end{aligned}
$$

in Eq. $2, \theta_{s} 、 \dot{\theta}_{s} 、 \ddot{\theta_{s}}$ is angular displacement, angular velocity and angular acceleration of gear (s); $T_{g}$ is the torque of the input of the cutting machine to the rocker reduction system; $T_{9}$ the torque on the rocker arm to the planet's head is the output torque of the shearer's rocker mechanism; $T_{s m}$ and $T_{m s}$ are the torque between gear $\mathrm{s}$ and gear $\mathrm{m}$, inside moment, they are in the opposite direction and is an unknown parameter, $F_{t}$ can be calculate by Eq. $3^{[9]}$ :

$$
F_{t}=\frac{2 T}{d}=\frac{2 T}{z m}
$$

In Eq. 3, $\mathrm{d}$ is standard pitch diameter of drive gear, $\mathrm{z}$ is gear tooth number, $\mathrm{m}$ is gear modulus. According to the relationship between acting force and reaction force, in the driving wheel and the 
driven wheel of the forces of equal size, direction is in the opposite, a pair of meshing gears between the roles of torque has the Eq. $4^{[10]}$ :

$$
T_{s m}=T_{m s} \cdot \frac{Z_{s}}{Z_{m}}=T_{m s} \cdot i_{s m}
$$

By Eq. 2 and Eq. 4, in accordance with the law of the matrix will be simplified and eliminated, we can derive the Eq. 5:

$$
\begin{aligned}
& \left(\begin{array}{c}
\ddot{\theta_{g}} \\
\ddot{\theta_{8}} \\
\ddot{\theta_{9}}
\end{array}\right)=\left(\begin{array}{ccc}
1 / J_{g} & 0 & 0 \\
0 & 1 /\left(i_{18} J_{1}+i_{28} J_{2}+i_{38} J_{3}+i_{48} J_{4}+i_{58} J_{5}+i_{68} J_{6}+i_{78} J_{7}+J_{8}\right) & 0 \\
0 & 0 & 1 / J_{9}
\end{array}\right) \\
& \left\{\left(\begin{array}{c}
T_{g} \\
0 \\
T_{9}
\end{array}\right)-\left(\begin{array}{cccc}
C_{g} & 0 & 0 \\
0 & i_{18} C_{1}+i_{28} C_{2}+i_{38} C_{3}+i_{48} C_{4}+i_{58} C_{5}+i_{68} C_{6}+i_{78} C_{7}+C_{8} & 0 \\
0 & 0 & C_{9} & \dot{\theta_{8}} \\
\dot{\theta_{9}}
\end{array}\right)-\left(\begin{array}{ccc}
K_{g 1} & -i_{18} K_{g 1} & 0 \\
-K_{g 1} & i_{18} K_{g 1}+K_{89} & -K_{89} \\
0 & -K_{89} & K_{89}
\end{array}\right)\left(\begin{array}{l}
\theta_{g} \\
\theta_{8} \\
\theta_{9}
\end{array}\right)\right\}(5
\end{aligned}
$$

\section{Mathematical model of planetary reduction system}

The transmission structure of the planetary reduction system of the cutting part of the cutting part of the large mining height drum shearer, it can be known that the planetary reduction system of the cutting part is composed of a $2 \mathrm{~K}-\mathrm{H}$ type planetary reduction gear mechanism, conduct force analysis, establish its mathematical model.

For the planetary reducer, the role of torque has the Eq. 6:

$$
\left\{\begin{array}{l}
J_{9} \ddot{\theta_{9}}+C_{9} \dot{\theta_{9}}=T_{i n}-T_{10} \\
J_{10} \ddot{\theta_{10}}=T_{10} \\
\left(J_{X 1}+J_{D 10-X 1} \ddot{\theta}_{X 1}+C_{X 1} \dot{\theta_{X 1}}+K_{X 1-12}\left(\theta_{X 1}-\theta_{12}\right)=T_{X 1}\right. \\
J_{R} \ddot{\theta}_{R}+C_{R} \dot{\theta_{R}}+K_{X 2 R}\left(\theta_{R}-\theta_{X 2}\right)=T_{\text {out }}
\end{array}\right.
$$

in Eq. 6,under the foot of standard X1、R said related parameters of retainer 1, rotary drum ; $J_{D 10-X 1}$ represents the moment of inertia of the 10 equivalent of the planet wheel to the retainer ; $T_{i n}$ is input torque of planetary reduction system of coal mining machine; $T_{\text {out }}$ is resistance moment of cutting coal in coal mining machine. According to relationship between power and torque as Eq. 7:

$$
T=9550 \cdot \frac{P}{n}
$$

In the $2 \mathrm{~K}-\mathrm{H}$ type planetary gear mechanism, the following drive ratio is Eq. 8,

$$
i_{9-11}^{X 1}=\frac{n_{9}^{X 1}}{n_{11}^{X 1}}=\frac{n_{9}-n_{X 1}}{n_{11}-n_{X 1}}
$$

\section{The drive system of the global dynamics simulation model}

The power and torque of the cutting machine is by the gear transmission system of the rocker arm is transmitted to the planetary gear system and the energy output is finally made by the roller. So the output torque of the gear 9 in the rocker reducer $T_{9}$ is the input torque of the planetary reduction system $T_{\text {in }}$, The final output torque $T_{\text {out }}$ of the planetary reduction system is the cutting torque $T_{R}$ of the drum of the drum shearer, by Eq. 5, Eq. 6, Eq. 7 and Eq. 8, the dynamic equation of the whole drive system of the cutting part can be obtained as Eq. 9, 


$$
\left(\begin{array}{c}
\ddot{\theta_{g}} \\
\ddot{\theta_{g}} \\
\ddot{\theta_{R}}
\end{array}\right)=\left(\begin{array}{ccc}
1 / J_{g} & 0 & 0 \\
0 & 1 / J_{9 e} & 0 \\
0 & 0 & 1 / J_{R}
\end{array}\right)\left\{\left(\begin{array}{c}
T_{g} \\
0 \\
T_{R}
\end{array}\right)-\left(\begin{array}{ccc}
C_{g} & 0 & 0 \\
0 & C_{9 e} & 0 \\
0 & 0 & C_{R}
\end{array}\right)\left(\begin{array}{c}
\dot{\theta_{g}} \\
\dot{\theta_{g}} \\
\dot{\theta_{R}}
\end{array}\right)-\left(\begin{array}{ccc}
K_{g 1} & -i_{19} K_{g 1} & 0 \\
-K_{g 1} & i_{19} K_{g 1}+i_{X 1-9} K_{X 1 R} & -K_{X 1 R} \\
0 & -i_{X 1-9} K_{X 1 R} & K_{X 1 R}
\end{array}\right)\left(\begin{array}{l}
\theta_{g} \\
\theta_{g} \\
\theta_{R}
\end{array}\right)\right\}
$$

\section{The dynamics simulation of the drive system}

In Figure 1 type double drum shearer as an example, in accordance with (11) type cutting part of the input shaft (cutting motor shaft) and drum of rotation angle acceleration, the change of angular velocity and angular displacement as shown in Fig.2.

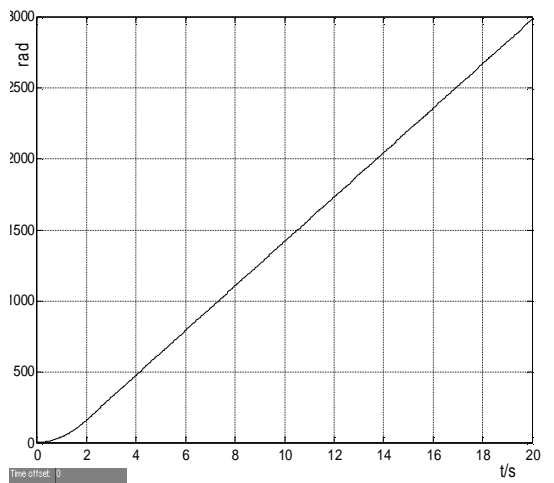

(a)Input shaft angle displacement

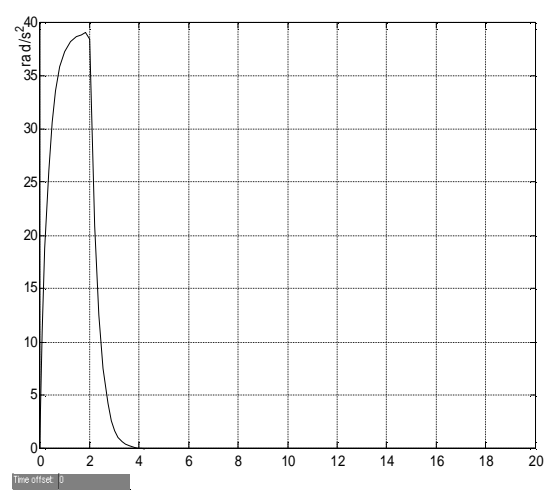

( d ) Angular velocity of drum

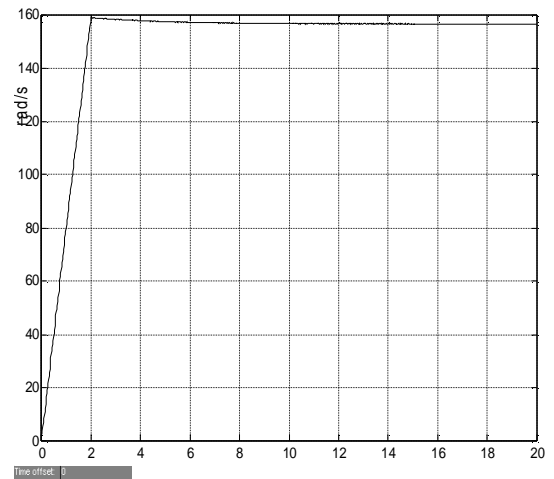

( b ) Input axis angular velocity

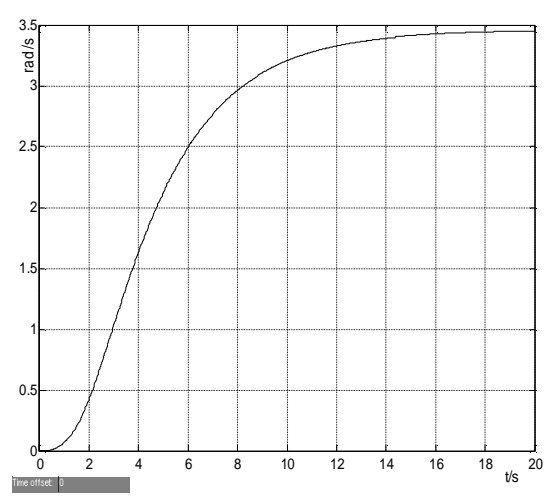

( e ) Input axis angular acceleration

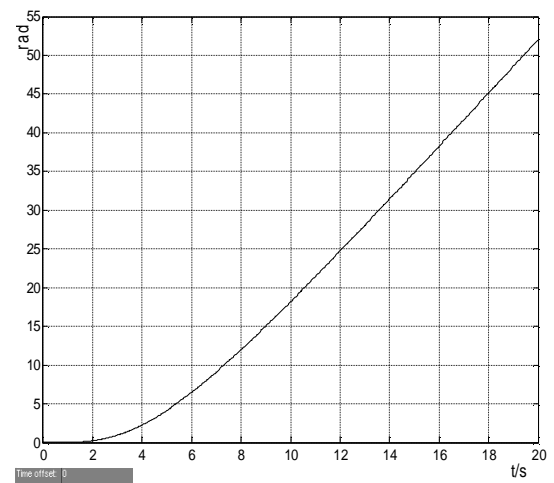

( c ) Angular displacement of drum

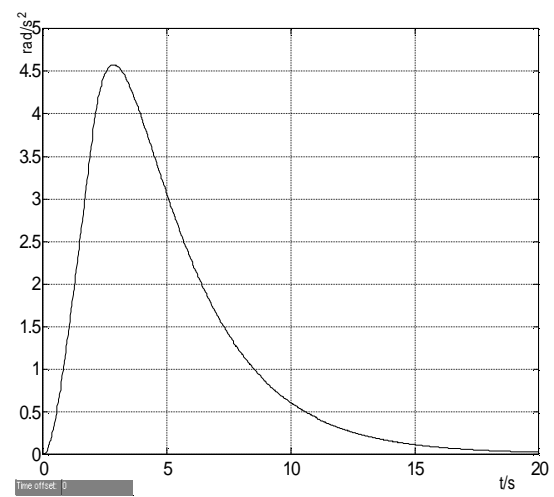

( f ) Roller angular acceleration

Fig.2 Dynamics simulation of the driving system of the cutting unit on idling

\section{Acknowledgment}

The authors would like to thank Prof. Zhaojian Yang and all the colleagues in Dept. of Automation Engineering in Shanxi University for their guidance, encouragement and advice.

\section{References}

[1] T.yang, "MG200/500-AWD、MXG-300/700 AC Electric Shearer Typical Mechanical Failure Analysis and Handling Way", Science and Technology of West China, 2008, vol.07, pp.11-12

[2] S.H.Ma, "Electric Shearer Common Mechanical Failure Analysis", Hydraulic Coal Mining \& Pipeline Transportation, 2008, vol.3, pp.74-75

[3] L.H.WANG, "Fault Analysis and Disposal Fault of Height Adjustment System in Electrical Traction Shearer”, Coal Mine Machinery, 2008, vol.29, pp.201-203

[4] W.H.Li, "MGTY-300/710 Electric Shearer Mechanical Failure Analysis and Handling Way", Hydraulic Coal Mining \& Pipeline Transportation, 2007, pp.(1)36-38 
[5] B.Y.FANG and G.B.YU, "Testing Method for Loading Cutting Part with High Power of Shearer", Coal Technology, 2006, vol.25, pp.3-5

[6] L.Z.LIU and L.P.HUANG, "Research and Manufacture About the Loading Test Bed for Shearer Cutting Part undergoing Maintenance”, Coal Technology, 2007, vol.26, pp.6-8

[7] Hu-xiang GENG. Usual Breakdown and Reason Analysis of MG571DWShear[J]. Coal Mine Machinery,2007(2):151-152. In Chinese

[8] Wen-guang SONG. Failure Analysis and Exclustion for Hydraulic Machinery of Shearer[J]. Coal Mine Machinery,2009(3):185-187. In Chinese

[9] Jie Meng, Xiaoan Chen. Transfer matrix method for dynamic analysis of the motorized spindle [J]. ournal of machine design,2008,25:37-40. In Chinese

[10] Kezhen Yang. Basics of Mechanical Design [M].Beijing: Higher Education Press,2007. 